\title{
Associations between gender, year of study and empathy level with attitudes towards animal welfare among undergraduate doctor of veterinary medicine students in Universiti Putra Malaysia.
}

\author{
Fazerin Azra Mohamed Azahar ${ }^{1}$, Nik Mohd Rizal Mohd Fakri ${ }^{2}$, Mohamad Najib Mat Pa ${ }^{2}$ \\ ${ }^{1}$ Master of Sciences Student, ${ }^{2}$ Department Medical Education, Universiti Sains Malaysia
}

\begin{tabular}{ll}
\hline ARTICLE INFO \\
Received & $: 05 / 10 / 2014$ \\
Accepted & $: 25 / 11 / 2014$ \\
Published & $: 01 / 12 / 2014$
\end{tabular}

\section{KEYWORD}

Gender

Year of study

Empathy

Animal welfare

Doctor of Veterinary

Medicine

\section{ABSTRACT}

Introduction: Attitudes towards animal welfare are important in influencing how animals are treated. Studies of attitudes towards animal welfare in veterinary students are scarce. It is hope that the findings will enhance a diverse research in the future in order to explore variety of factors in relation to animal welfare since such study is currently limited. Objective: The study is to determine the associations of gender, year of study and empathy level of undergraduate DVM students in UPM with their attitudes towards animal welfare. Method: Questionnaires were given to 440 Doctor of Veterinary Medicine undergraduate students in Universiti Putra Malaysia to study the association between gender, year of study and empathy level with attitudes towards animal welfare. Data were collected from respondents through two sets of self-guided questionnaires; Interpersonal Reactivity Index (IRI) which assessed empathy level where only two sub-scales from the IRI were used; Empathic Concern (EC) and Perspective Taking (PT). Animal Attitude Scales (AAS) were used to assess attitudes towards animal welfare. Data collected were analysed using Statistical Package for Social Science (SPSS) version 20. Result: 367 (83.4 $\%)$ out of 440 students participated in this study. Anti-animal welfare attitude (74.9\%) was the highest compared to the pro-animal welfare attitude (25.1\%). Analysis showed a significant difference $(\mathrm{p}<0.05)$ between year of study and attitudes towards animal welfare $(p=0.001)$, however, there was no significant differences $(p>0.05)$ between gender and attitudes towards animal welfare $(p=0.057)$ as well as between empathy level and attitudes towards animal welfare for the empathic concern subscale $(p=0.194)$ and perspective taking sub-scale $(p=0.320)$. Conclusion: Majority of students were categorized as anti-animal welfare and the attitude were significantly different among years of study. Female and male students have no significant difference in their attitudes towards animal welfare. Students with good and poor empathy level also have no significant difference in their attitudes towards animal welfare.

(c) Medical Education Department, School of Medical Sciences, Universiti Sains Malaysia. All rights reserved.

CORRESPONDING AUTHOR: Dr. Fazerin Azra Mohamed Azahar, Master of Sciences Student, Department Medical Education, Universiti Sains Malaysia. Email: fazerin_azra@yahoo.com 


\section{Introduction}

Animal welfare is the avoidance of abuse and exploitation of animals by humans. This global issue becomes worrying each day, although incessant efforts and funding have been contributed to educate public on animal welfare, prevention and control against animal cruelty. It is of no doubt with the various animal rights campaigns have been an increase research on attitudes toward animals (1-3). Studies have shown correlation between level of empathy and attitudes of individual towards animal welfare (4, 2).

The increased research pertaining to animal welfare over the years reflects the growing concern of people. In Malaysia, RM80 million is allocated for improving animal welfare through its National Animal Welfare Strategic Plan 20122020 (NAWSP). The aim of the plan is to cultivate responsible animal owner with provision of education and training in animal welfare (5). Among the important people that need education and training in animal welfare are the Doctor of Veterinary Medicine (DVM) undergraduates since they work closely with animals and they are obliged to promote good animal welfare. Therefore investigation of the level of empathy and attitudes of the students is crucial.

It is assumed that those involved in the veterinary profession would have highest standards of animal welfare attitudes (6). A study by Paul \& Podberscek (3) had proven otherwise where degradation of perception on animal welfare occurs among students towards their later years of study and males were found to have lower levels of empathy towards animals than did the females. However, the level of empathy and attitudes of our veterinary students in Malaysia is unknown which makes this study quite relevant and significant.

\section{Method}

Questionnaires were given to 440 Doctor of Veterinary Medicine undergraduate students in Universiti Putra Malaysia to study the association between gender, year of study and empathy level with attitudes towards animal welfare. Data were collected from respondents through two sets of self-guided questionnaires; Interpersonal Reactivity Index (IRI) which assessed empathy level where only two subscales from the IRI which were Empathic Concern (EC) and Perspective Taking (PT). Animal Attitude Scales (AAS) were used to assess attitudes towards animal welfare.

Data collected were analysed using Statistical Package for Social Science (SPSS) version 20.The three factors were analysed using Pearson Chi Square because independent variables (gender, year of study and empathy level) and dependent variables (pro-animal welfare or antianimal welfare) were in the categorical forms. The alpha level was set at $\mathrm{p}<0.05$ for each hypothesis.

The distribution of total attitudes and empathy level scores were examined separately for normality in order to determine the cut-off score which will help to classify students' attitudes towards animal welfare either as pro or anti. Score distribution is considered normal if the skewness is between -1 and +1 (7).

If the attitudes and empathy scores are normally distributed, the mean score of attitudes and empathy will be taken as a cut-off score for pro or anti-animal welfare and good or poor empathy level. If the attitudes and empathy scores are not normally distributed, the median will be taken as a cut-off score.

Ethical approval is required to ensure that the study meets the criteria of ethical standards; hence, ethical clearance was obtained from the school (School of Medical Sciences, Universiti Sains Malaysia) and ethical committees prior to the start of the study. Participants' anonymity and confidentiality were preserved and maintained. The researcher explained clearly in the consent form regarding the purposes of this study, methods of data collection and risk if they participate in this study. The formal signature of consent was obtained from each participant who agreed to participate in the study. 


\section{Result}

There were 367 (83.4\%) out of 440 students participated in this study. Anti-animal welfare attitude (74.9\%) was the highest compared to the pro-animal welfare attitude (25.1\%). Analysis showed a significant difference $(\mathrm{p}<0.05)$ between year of study and attitudes towards animal welfare $(p=0.001)$, however, there was no significant differences ( $\mathrm{p}>0.05$ ) between gender and attitudes towards animal welfare $(\mathrm{p}=$ 0.057 ) as well as between empathy level and attitudes towards animal welfare for the empathic concern sub-scale $(\mathrm{p}=0.194)$ and perspective taking sub-scale $(p=0.320)$ (see Table 1).

Table 1: Summary of associations between gender, year of study and empathy level with attitudes towards animal welfare

\begin{tabular}{|c|c|c|c|c|}
\hline \multirow{2}{*}{ Factor } & & \multicolumn{2}{|c|}{ Attitudes towards animal welfare, $\mathbf{n}(\%)$} & \multirow{2}{*}{$p$ value } \\
\hline & & Pro-animal welfare & Anti-animal welfare & \\
\hline \multirow[t]{2}{*}{ Gender } & Male & $16(17.6)$ & $75(82.4)$ & \multirow{2}{*}{0.057} \\
\hline & Female & $76(27.5)$ & $200(72.5)$ & \\
\hline \multirow[t]{5}{*}{ Year of study } & 1 & $11(14.1)$ & $67(85.9)$ & \multirow{5}{*}{$* 0.001$} \\
\hline & 2 & $24(23.5)$ & $78(76.5)$ & \\
\hline & 3 & $13(18.6)$ & $57(81.4)$ & \\
\hline & 4 & $23(33.3)$ & $46(66.7)$ & \\
\hline & 5 & $21(43.8)$ & $27(56.2)$ & \\
\hline \multirow[t]{2}{*}{ Empathic Concern (EC) } & Good & $146(72.3)$ & $56(27.7)$ & \multirow{2}{*}{0.194} \\
\hline & Poor & $129(78.2)$ & $36(21.8)$ & \\
\hline \multirow[t]{2}{*}{ Perspective Taking (PT) } & Good & $142(72.8)$ & $53(27.2)$ & \multirow{2}{*}{0.320} \\
\hline & Poor & $133(77.3)$ & 39 (22.7) & \\
\hline
\end{tabular}

${ }^{*} \mathrm{p}<0.05$ is considered as significant

\section{Discussion}

The study conducted among undergraduate DVM students was to learn the associations of gender, year of study and empathy level on attitudes towards animal welfare. The concern stems from which attitudes towards animal can predict on how one's behave or perceived animals (8). It is anticipated that veterinary students whom will be the future veterinarian should have positive attitudes towards animal. At certain degree, they should at their best promote good behaviour and practice towards animals. The factors studied are discussed separately in the following sections.

\section{Attitudes towards animal welfare according to gender}

Gender is the most prevalent variable that is related generally to animal welfare attitudes (9). Gender has been suggested to affect attitudes towards the treatment of animals (10).

It was expected that the DVM students would display higher number in the pro-animal welfare category. Unexpectedly it has proven otherwise where majority of students were categorized in anti-animal welfare. Nevertheless, female students have outperformed the male students in the pro-animal welfare attitudes category. The probable cause of current study on gender turned out the opposite way might due to the under representativeness of the male respondent; male to female ratio 1:3.

This particular finding is consistent with the literatures $(2,11,12)$ where females are known to have positive attitudes towards animal welfare. It has been suggested that this difference of attitudes maybe associated with women being more "tender-minded" than men (13) which leads them to be more concerned about issues involving pain and death.

A few researchers that utilized AAS in their studies found that women of psychology undergraduate generally had more positive attitudes to animals than did the men (2). Women were found always to be more sympathetic to the treatment of animals than were men (11). Signal \& Taylor (14) found that females from both 
groups of animal protection groups and general community in Australia have more positive attitudes. Likewise, in a study related to animal welfare among British undergraduates, women students were higher in number than men who were against animal testing (15).

However, the percentage of female students in the anti-animal welfare category is alarming which is not consistent with previous studies where many have found that women are more likely to support the animal rights movement and to oppose animal research than men (16-20). The current finding has caused a stir where one would ask what had happened to these "tenderminded" (female students) group? Is it because of their learning environment or different cultural background or personality or stressful learning culture have hardened them?

This finding may reflect that male and female shares similar perceptions and attitudes towards animal in terms of their welfare even if they perceive negatively on animal welfare issues. Although, this factor is not significantly associated with attitudes towards animal welfare $(p=0.057)$, it does not mean that this could conform to related study in the future of the local population where the subjects are the veterinary students.

This current finding warrants serious investigation that may suggest some interventions for the students that could curb the situation from continuously deteriorating because further negligence may result in majority females would be categorized in antianimal welfare rather the male counterparts or both gender fiercely competing in the negative attitude.

\section{Attitudes towards animal welfare according to year of study}

Majority of the students regardless of their year of studies have anti-animal welfare attitude. The trend of students with pro-attitudes towards animal welfare is not stable because it fluctuated over the year of studies. However, year four to year five showed a significant regain of the proanimal welfare attitude. On contrary, anti-animal welfare attitude showed significant decline from fourth year onwards.

Does this indicate attitude towards animal welfare improve towards later year of study? Does the improvement resemble the effectiveness of the learning environment at the faculty? The questions could be further proven if a longitudinal study will be conducted in the future among the DVM students at UPM because we cannot confirm the early discovery with later finding.

What had happened to the attitudes of the second year DVM students towards animal welfare? The question brought up because Veterinary Ethics, Animal Welfare and Jurisprudence were taught during second year at FVM, UPM. It was expected that second year students could be the champion of being pro-animal welfare among other year of study; however, it appeared the opposite.

This discovery could notify the faculty to further assess the related subject to animal welfare taught to the second year students. Nevertheless, the findings have illustrated that positive attitude towards animal welfare increased in proportion to the increase in workload of the clinical year students by attending animal patients or cases.

The finding contradicts from previous studies done among veterinary undergraduates $(3,21)$ where students' attitudes have faced a gradual declination in their later years. However, the latter studies were conducted among students from certain year of study for example from the beginning of first year until end of first year unlike the current study where students of all year of studies were included.

Hence, there still a room to argue on the pattern of attitudes throughout the year of studies in veterinary program and again, this factor warns consideration for further investigation in order to predict at which year does students start to behave positively or vice versa towards animal be it animal welfare or animal use.

\section{Attitudes towards animal welfare according to empathy level}

Empathy level was found to have no significant difference in attitudes towards animal welfare (EC; $p=0.194$, PT; $p=0.320$ ). Those with good and poor empathy level in both sub-scales (EC and PT) obtained close scores; 63.36 vs. 62.52 and 63.33 vs. 62.59 respectively.

Although, empathy level (EC and PT) is not significantly correlated with attitudes towards animal welfare, EC shows significant association 
( $\mathrm{p}=0.003)$ with gender whereby PT was poorly associated with gender $(\mathrm{p}=0.569)$.

This finding is further supported by Taylor \& Signal (22) who utilised IRI and found a significant correlation between empathic concern and attitudes towards animals in a sample of undergraduate sociology and psychology students. Females scored more positively on attitudes towards animals than did males. Other finding proved that EC and PT were significantly related to attitudes towards animal use in medical and psychological research (4).

Previous studies done (3) have discovered that males were found to have lower levels of empathy towards animals than did the females on a study done among veterinary students in United Kingdom.

A study done (9) on public perceptions of animal welfare revealed that a variable that is also correlated with gender is empathy with women generally showing higher scores on empathy than men. General findings of empathy level have gender bias' against males, this is probably because male brains are being characterized by the systemizing (23) that is the drive to analyze, understand and predict the law-governed nonliving universe as well as the ability to analyze changing patterns, to figure out how things work. On the other hand, female brains were mostly being driven by the empathizing which is the drive to identify another person's emotions and thoughts (24).

The current study found that year of studies was poorly associated with empathy (EC; $p=0.707$ and PT; $\mathrm{p}=0.320$ ). However, majority of students regardless of their year of study favour good empathy level in both sub-scales.

It is anticipated that level of empathy would degrade as discovered before (21), first year of veterinary undergraduate students have decreased level of empathy in their first year of study. In another study, declination of empathy level of undergraduate medical students towards patients occurs from third year of study onwards (26).

However, it is not sufficed to assume the exact pattern at which year does the undergraduate of DVM student start to degrade their empathy or at which year does the empathy regain. Results pertaining to empathy level appear differently from previous studies may be due to some limitations by the use of truncated IRI where it may neglect other important interactions between human empathy and attitudes towards the treatment of animals (14).

Empathic concern (EC) sub-scale has been found to relate to global measures of emotion in which it reflects a general concern for others (25). EC appears to have strong relationships with AAS most probably because it taps into animaldirected empathy as well as human-directed empathy (14).

Perspective taking (PT) relates to general and interpersonal social functioning (25). Some may find the association between PT and AAS to be less strong may be due to its ability to take other's view who favour more functional attitude to the treatment of animals. For example, respondents from animal rights and animal welfare society have different perspective on animal rights or animal welfare issues compared to those who are non-member therefore, it is expected that the outcome on PT will differ greatly and chances are the animal rights members will get higher scores.

\section{Strengths of the study}

The strengths of the study rely on the questionnaires that were used (IRI and AAS). Each item in the questionnaire was originally constructed in simple and familiar words in English. Moreover, English is a spoken language at FVM. Therefore, no alterations were made to both of the questionnaires. This helps in reducing the length of time required to answer the questionnaire, which is why the questionnaire only takes less than 10 minutes upon completion.

The number of items contained in both questionnaires (IRI and AAS) also played important role that could give impact to the result. There are 20 items in AAS, seven items in each sub-scale of IRI that makes 34 items to be answered by respondent in total. Both questionnaires are considered feasible because of its questions are simple, precise and adequate in number (not less than 20 and not more than 40 items) although there are no universal agreements about the optimal length of questionnaires (27). This is because, lengthy and over extended questionnaires could cause boredom or exhaustion (28) to respondent which will implicate their answers. 


\section{Limitations of the study}

One of the limitations identified was sample size determination. The students were sampled based on convenience sampling in which all of the students of year one to year five who were eligible to participate were included in this study. This is because the limited number of reference from previous studies that were done to DVM undergraduate students especially among local population using the same set of questionnaires with the current study. There were limited studies on the effects of year of study and empathy level of local undergraduate DVM students in particular. Hence, it provides little information on the ideal number of sample size. However, based on previous studies $(2,12)$, they also applied convenience sampling method where the results were achievable and acceptable

When discussing animal welfare in Malaysia, it is important to point out that animal welfare, as a science, originated in the West and it developed alongside with scientific and technological advances (29). Moreover, cultural differences between East and West, as the Eastern likeness toward the natural world is actually highly restricted because of respect and appreciation and these are often restricted to particular species (29). For example, Hindhus (especially in India) show high respect to cattle because of its sacredness according to their religion, hence, they will not agree to any activities that would harm the species but what about other religions that consume beef from cattle? The answer scripts of both different groups will appear totally different perhaps.

The used of truncated version of IRI questionnaire suggested some limitations where it may overlook other important interactions as what have been discussed earlier. Besides that, some of the items in AAS could also have little importance and attention among local population, such as the issue on whale and dolphin slaughter, the use of animal in rodeos and circus. These particular issues were not a common practice in Malaysia. Furthermore, whale and dolphin do not inhabit (or commonly migrating to) our coastal area and they are not originated here. The rodeos and circus is also not commonly practiced in our culture. Rodeos are synonym among cowboys of western countries like America. Therefore, these items will display inconsistent consensus among the DVM students.
The other limitation pertaining to questionnaire was the scoring system where the cut-off scores for AAS and IRI were based on norm reference which is highly dependent on population. This is because a standardized base line or cut-off score have not yet achieved which is why authors of both questionnaires encouraged the utilization of their questionnaires in this kind of study so that in the future, they will come out with a standardized cut-off score.

\section{Recommendations}

I would highly suggest that a new set of questionnaire to study the attitudes towards animal welfare could be constructed that would be suitable to our culture and surrounding nature. The idea is to conduct a study focusing on local environment first then along with incessant animal welfare campaign and activities, a broader scope of animal welfare issues could be studied in the future.

It would be a good starting point to conduct a similar study in the future with a larger sample size of veterinary students perhaps since the kind of study done locally is limited. This is because a large sample size could support with an adequate baseline data in this study of investigation (14).

This study did not measure the effect of affiliation to animal rights or welfare society where the percentage of non-member was 96 percents out of total students participated. This huge portion might have influenced the findings in which it has caused the scores of each questionnaire to differ from other findings done internationally; hence, this factor should be studied in the future to determine its association with attitudes towards animal welfare.

The other possible aspect that might have influence students' attitudes towards animal welfare is residential area. The distance of their residents might be far away from the zoo, animal centre, animal shelter, farms or other places that could provide them exposure to animals.

Presence of companion animal (pet) could possibly have affected the finding of current study because 272 (74.1\%) of the students participated claimed to own a pet/ pets. It is suggested that those who own pet(s) would portray positive attitude towards animal welfare, however, the current findings showed majority of the students were negative towards animal welfare. This factor should be investigated 
further in order to discover the significant association with attitudes towards animal welfare.

Vegetarianism has been reported to have a significant role on attitudes towards animal welfare (15). Over 350 (97\%) of the DVM students who responded in this study were not vegetarianism. Could it be non-vegetarianism contribute to highest number of students in antianimal welfare category? It is understood that vegetarian avoid consuming or do not consume meat or animal product in which it reflect that they oppose animal pain and suffering but are they? This factor deserves an empirical study in the future.

Other demographic factors that have the possibility to influence the attitudes of respondents towards animal welfare are difference culture and religion background as well as personality and stress level. However, effects of the demographic factors (besides gender, year of study and empathy level) on the study were derived from assumptions in which they need to be quantified for credible findings to support. Hence, this area warrants further investigation in order to determine predictive factor upon empathy level. In addition to that, the utilising of other empathy scales is encouraged in order to provide baseline data from various sources on local population's attitudes towards animal welfare.

The current discoveries on attitudes of DVM students towards animal welfare should draw attention to the faculty to plan and conduct appropriate program(s) for the students that could enhance students' attitudes toward animal welfare by giving them more exposure on local and international issues of animal welfare. It would be meaningful if hands-on activities such as performing proper method and procedure of handling patient were offered during the course. To further appreciate knowledge and skills on Veterinary Ethics and Animal Welfare that are taught at the faculty, students should be encouraged to involve in volunteer project or internship program at appropriate places that offer them a handful of job experience attending animals other than courses that are taught in the curriculum.

Furthermore, the level of awareness regarding animal welfare can be determined through intervention (before and after intervention) where it helps the faculty to evaluate the activities or structured curriculum provided to the students in order to educate them on animal welfare. Shankar \& Priyani (2013) have found positive changes in empathy among first year medical students before and after a medical humanities module was introduced to them.

Regardless of these informative findings, more diverse research pertaining to animal welfare particularly related to undergraduate DVM students should be carried out in the future in order to extend the findings of predictive factors that are associated with attitudes towards animal welfare and it may likely leads to other valuable discovery.

\section{Conclusion}

Majority of students were categorized as antianimal welfare and the attitude were significantly different among years of study. Female and male students have no significant difference in their attitudes towards animal welfare, however, female students were the majority in the pro-animal welfare category. Students with good and poor empathy level also have no significant difference in their attitudes towards animal welfare. Nevertheless, the number of students who possess good empathy level outnumbered those with poor empathy level.

\section{References}

1. Coilee D, Miller N. How radical animal activity try to mislead humane people. American Psychologist, 1984; 39: 700-701.

2. Matthews S, Herzog HAJ. Personality and Attitudes toward the Treatment of Animals. Society and Animals, 1997; 5: 169-175.

3. Paul E, Podberscek A. Veterinary education and students' attitudes towards animal welfare. Animal Welfare, 2000; 13: S145S151.

4. Furnham A, McManus C. Scott D. Personality, empathy and attitudes to animal welfare. Anthrozoos, 2003; 16(1): 135-146.

5. Government to spend RM 80 million to boost animal welfare, New Straits Times (November $7^{\text {th }}, 2012$ ), pp 2. Homepage [online] available from http://www.nst.com.my/nation/general/govtto-spend-rm80m-to-boost-animal-welfare1.167728\#ixzz2CAgpDuj6 [accessed November 8 2012].

6. Levine ED, Mills DS, Houpt KA. Attitudes of Veterinary Students at One US College 
toward Factors Relating to Farm Animal Welfare. Journal of Veterinary Medicine Education, 2005; 32(4): 481-490.

7. Morgan GA, Griego OV, Gloeckner GW. SPSS for Windows: An introduction to use and interpretation in research. Lawrence Erbaum Associates Publisher, 2001.

8. Azjen I, Fishbein M. Understanding Attitudes and Predicting Social Behaviour. Englewood Cliffs, NJ: Prentice Hall, 1980.

9. Coleman G. Public attitudes to animal research. Proceedings of the ANZCCART conference, Christchurch NZ, 18- 19 August 2003, 78-86.

10. Herzog HAJ, Betchart NS, Pittman R. Sex role identity and attitudes toward animals. Anthrozoos, 1991; 43(3): 184-192.

11. Herzog, H. A. Jr. (2007). Gender differences in human- animal interactions: A review. Anthrozoos; 20(1): 7-21.

12. Signal TD, Taylor N. Attitudes to animals within a community sample. Society and Animals, 2005; 14(2): 147-157.

13. Broida L, Tingley L, Kimball R, Miele R. Personality differences between pro- and anti-vivisectionists. Society and Animals, 1993; 1: 129-144.

14. Signal TD, Taylor N. Attitude to animals and empathy: comparing animal protection and general community samples. Anthrozoos, 2007; 20(2): 125-130.

15. Furnham A, Pinder A. Young people's attitude to experimentation of animals. Psychologist, 1990; 10: 444-446.

16. Bailey M. Women and support for the animal rights movement, 1948-1985. Paper presented at the meeting of the Midwest Political Science Association, Chicago, IL, 1994.

17. Gallup GG, Backstead JW. Attitudes toward animal research. American Psychologist, 1988; 43: 474-476.

18. Galvin SL, Herzog HAJ. Ethical ideology, animal rights activism and attitudes toward the treatment of animals. Ethics and Behaviour, 1992; 2: 141-149.

19. Kellert SR, Berry JK. Attitudes, knowledge and behaviours towards wildlife as affected by gender. Wildlife Society Bulletin, 1987; 15: 363-371.

20. Plous S. An attitude survey of animal rights activists. Psychological Science, 1991; 2: 194-199.

21. Nunes N., WilliamsS, Sa B, Stevenson K. A study of empathy decline in students from five health disciplines during their first year of training. Journal of Medical Education, 2011; 2: 12-17.

22. Taylor N, Signal T. Empathy and attitudes towards animals. Anthrozoos, 2005; 18(1): 18-27.

23. Baron-Cohen S, Richler J, Bisarya D, Gurunathan N, Wheelwright S. The Systemizing Quotient: An investigation of adults with Asperger syndrome or high functioning autism and normal sex differences. Philosophical Transactions of the Royal Society, 2003; 358: 361-374.

24. Baron-Cohen S. Zero Degrees of empathy. A New Theory of Human Cruelty. London: Penguin Books, 2011.

25. Davis MH. A multidimensional approach to individual differences in empathy. JSAS Catalogue of Selected Documents in Psychology, 1980; 10: 85.

26. Hojat M, Vegare MJ, Maxwell K, Brainard, G, Herrine SK, Isenberg GA, Veloski J, Gonella S. The Devil is in the Third Year: A Longitudinal Study of Erosion of Empathy in Medical School. Academic Medicine, 2009; 84: 1182-1191.

27. Leung WC. How to design a questionnaire? Student Biomedical Journal, 2001; 9: 187189.

28. Krosnick JA, Presser S. Question and Questionnaire Design. Handbook of Survey Research. Second Edition, (Eds). Wright, J. D. and Marsden, P. V., San Diego, CA: Elsevier, 2009, pp 1-81.

29. Davey G. Chinese University Students' Attitudes toward the Ethical Treatment and Welfare of Animals. Journal of Applied Animal Welfare Science, 2006; 9(4): 289297.

30. Shankar PR, Piryani RM. Changes in empathy among first year medical students before and after a medical humanities module. Education in Medicine Journal, 2013; 5(1): 35-42. 